\title{
Bifurcation Thresholds in an SIR Model with Information-Dependent Vaccination
}

\author{
A. d'Onofrio ${ }^{a}$, P. Manfredi ${ }^{b}$ and E. Salinelli ${ }^{1}$ \\ ${ }^{a}$ Division of Epidemiology and Biostatistics, European Institute of Oncology \\ Via Ripamonti 435, 20141 Milano, Italy \\ ${ }^{b}$ Dipartimento di Statistica e Matematica Applicata all'Economia, Università di Pisa \\ Via Ridolfi 10, 5612 Pisa, Italy \\ ${ }^{c}$ Dipartimento di Scienze Economiche e Metodi Quantitativi, Università del Piemonte Orientale \\ “A. Avogadro", Via Perrone 18, 28100 Novara, Italy

\begin{abstract}
Simple epidemiological models with information dependent vaccination functions can generate sustained oscillations via Hopf bifurcation of the endemic state. The onset of these oscillations depend on the shape of the vaccination function. A "global" approach is used to characterize the instability condition and identify classes of functions that always lead to stability/instability. The analysis allows the identification of an analytically determined "threshold vaccination function" having a simple interpretation: coverage functions lying always above the threshold always lead to oscillations, whereas coverage functions always below never lead to instability.
\end{abstract}

Key words: SIR epidemiological models, information-dependent vaccination, stability, Hopf bifurcations

AMS subject classification: 92D30

\section{Introduction and main results}

A critical factor in determining the success of a vaccination programme is the "human element", i.e. the degree of social adhesion by individuals to the proposed vaccination. The history of vaccination documents indeed that the path toward increasing degrees of diseases control, for instance

${ }^{1}$ Corresponding author. E-mail: alberto.donofrio@ieo.it 
in the case of smallpox, has not been "linear" but rather constellated by episodes of coverage upswing arising from the tension between public health targets and individual freedom, i.e. between compulsory vaccination and conscientious or philosophical exemption [11]. In very recent times the dramatic decline in coverage of the Measles-Mumps-Rubella vaccine (MMR) in the UK (CDR 2004) has been explained by the reaction of the public to the adverse publicity about possible links between the vaccine, autism, and Crohn's disease [15], similar to what happened few years before to the HBV coverage due to the "Thimerosal" case [6].

These phenomena confirm that the public is not a passive actor of the vaccination process but that rather can feedback on it with far reaching implications. This fact is strongly emphasized by the vaccination success in controlling diseases, which encourages forms of "rational exemption". The argument goes as follows. Consider the example of measles control. In several countries the increasing measles coverage within the WHO Plan for measles eradication has driven circulation of the disease to minimal levels. As the incidence of the disease continues to decline thanks to vaccination, families become increasingly concerned with the risks associated with vaccines [14]. If families start to perceive that the chance of acquiring infection for their children is lower compared to the risk of experiencing damages from the vaccine, they could believe it "rational" not to vaccinate their children. This "rationality" is of course myopic. Moreover, it is an example of "free riding" [12], as by the way all types of exemptions [11].

In presence of a widespread adoption of rational exemption at least a part of families relate their decision to vaccinate to the available information on the state of the disease, vaccinating more, and promptly, when the "perceived" risk from the disease is high, and little (and later) otherwise.

From the theoretical point of view an important question deals with the implications of individuals' vaccination choice for the dynamics and control of vaccine preventable diseases. This issue has been considered in [10] and [2]. Though starting from a different background, i.e. game theory in order to fully embed individuals' behaviour [10], rather than a phenomenological function relating the response in terms of overall vaccination coverage to some information function describing the changes in epidemiological conditions over time [2], these papers come to the same formulation of a Susceptible-Infective-Removed model with vaccination in which the vaccination coverage is related to some function of the state of the disease.

As main dynamic result both studies have shown that the presence of delayed informationdependent vaccination, i.e. individuals who adapt their vaccinating behaviour to changing epidemiological conditions using not only the current but also the past available information on the state of the disease, can destabilise the endemic state of the SIR model with vaccination, leading to steady oscillations through Hopf bifurcations. In addition it was shown in [2] that these oscillations have a period which can be significantly longer compared to the predictions of the basic SIR model, a fact which must be carefully taken into account when designing control programmes.

It is therefore interesting to characterise which shapes of the function describing the reaction in individual's propensity to vaccinate to changing epidemiological conditions are more/less favourable to instability. The standard tools, i.e. local stability analysis, are not very informative from this point of view in that are highly dependent on the specific functional form chosen for the vaccination function at the endemic state. For this reason in this paper we resort to a different approach having a "global", rather than local, nature, i.e. we look for the set of coverage functions 
that, whatever is the value of endemic state, assure the instability or the stability of the endemic equilibrium. In other words our idea is to find a general criterion capable to establish a-priori if a given vaccination function is a potential generator of instability and oscillations. The only manner to do this is to reformulate the instability condition in a way that allows to evaluate the "pure" role of the vaccination function on stability, i.e. its role independently on the other parameters of the system. This is clearly not possible by working on the local stability condition where by definition the vaccination function and the remaining system parameters are complexly entangled. This dependency can however be broken down by resorting to a global approach. Our approach leads to a differential inequality. The study of such inequality allows the identification of an analytically determined "threshold function" having a simple interpretation: coverage functions lying always above the threshold function always lead to oscillations, whereas coverage functions lying always below the threshold function never lead to instability. The approach is illustrated by some examples using classical functional forms for the vaccination function. The examples show that the approach is quite promising in that it allows to well discriminate between the classes of functions that in concrete cases lead to stability or instability.

Finally, we would stress that stability problems similar those we studied here may arise in mathematical epidemiology whenever an epidemic phenomenon is modelled by means of a family of models containing one (or more) parameter expressed as a partially unspecified function $f$ of the state of the system, for which only some qualitative properties are known, such as continuity, monotonicity etc. As a consequence, the determination of equilibrium points depends on the shape of this function and the conditions guaranteeing their stability also involve the derivatives of $f$. For example, in [5] a family of models describing the spread of diseases in which the latency time depends on the infective load was proposed. In such model, the latent period is loosely defined as a decreasing function of $I$ and a condition for the stability of the endemic state is derived, which is conceptually similar to the one we studied here, and thus might be analysed by following the methodology proposed here.

The paper is organised as follows. In section 2 we present the model and report its main results. In section 3 we study the condition for the Hopf bifurcation of the endemic state via our "global" approach. Illustrative examples are reported in section 4. Concluding remarks follow.

\section{The model and its equilibria}

In [2] the following family of SIR models for a non-fatal disease in a constant homogeneously mixing population, with information-dependent vaccination coverage was introduced:

$$
\begin{aligned}
X^{\prime} & =\mu N(1-p(M))-\mu X-\beta \frac{X Y}{N} \\
Y^{\prime} & =\beta \frac{X Y}{N}-(\mu+\nu) Y \\
Z^{\prime} & =\nu Y-\mu Z \\
V^{\prime} & =\mu N p(M)-\mu V
\end{aligned}
$$


Variables $X, Y, Z, V$ are functions of time denoting respectively the number of susceptibles, infectious (and infectives), immune and vaccinated individuals at time $t$. Moreover, $\mu \in \mathbb{R}_{+}$ denotes the birth and death rate, which are assumed identical, $\nu \in \mathbb{R}_{+}$the rate of recovery from infection, $\beta \in \mathbb{R}_{+}$the transmission rate, and $N=X+Y+Z+V$ is the total population, constant over time. Thus it is useful to introduce the epidemiological fractions, i.e. the variables

$$
S=X / N, \quad I=Y / N, \quad R=Z / N, \quad U=V / N .
$$

The information variable $M$, summarizing information about present and past values of state variables, is expressed by an exponentially fading delaying memory (see [8]) of the form

$$
M(t)=\int_{-\infty}^{t} k I(\tau) a e^{-a(t-\tau)} d \tau \quad a, k \in \mathbb{R}_{+} .
$$

Note that by (2.3) it follows that $M$ is bounded, taking all the values of an interval $\mathcal{I}=\left[M(0), M^{\text {sup }}\right)$, where $M^{\text {sup }}=\sup _{t} M(t)$.

The coverage function $p$ denotes, assuming a $100 \%$ effective vaccine, the actual vaccination coverage at birth in dependence on $M$ defined as:

$$
p(M)=p_{0}+p_{1}(M) \quad 0<p_{0}<1, M \in \mathcal{I}
$$

where the fixed component $p_{0}$ captures the presence of a fraction of the population that vaccinates its children whatever be the state of publicly available information $M$. It seems reasonable to introduce the following assumptions (discussed in [2]):

A1) $\quad 0 \leq p_{1}(M) \leq 1-p_{0}$ for all $M \in \mathcal{I}$, with $p_{1}^{\text {sat }}=p_{1}\left(M^{\text {sup }}\right)$;

A2) $\quad p_{1}(0)=0$;

A3) $p_{1}$ is continuous and differentiable, except, in some cases, at a finite number of points, with $p^{\prime}(M) \geq 0$.

Briefly our assumptions (3)-(4) state that, while taking their decision to vaccinate or not their children, individuals use current and past information on a linear function of the standardised prevalence of infection. Such a function could represent for instance the incidence of serious cases of the diseases (but many other forms can be used, as discussed in [2]).

Combining (2.1), (2.2) and (2.3), our model is:

$$
\begin{aligned}
& S^{\prime}=\mu\left(1-p_{0}-p_{1}(M)\right)-\mu S-\beta S I \\
& I^{\prime}=I(\beta S-(\mu+\nu)) \\
& M^{\prime}=a(k I-M)
\end{aligned}
$$

where we have discarded variables $R$ and $U$ because their dynamics follow trivially from the dynamics of $S, I$ and $M$. 
System (2.5) always admits the disease-free equilibrium (DFE)

$$
D F E=\left(1-p_{0}, 0,0\right) \text {. }
$$

Under condition $\left(1-p_{0}\right) R_{0} \leq 1$, where $R_{0}=\beta /(\mu+\nu)$ denotes the Basic Reproduction Number of the Disease, the $D F E$ is globally asymptotically stable (GAS) (see [2]).

Under condition

$$
\left(1-p_{0}\right) R_{0}>1
$$

the unique endemic equilibrium $E E=\left(S_{e}, I_{e}, M_{e}\right)$ exists for (2.5), where

$$
S_{e}=\frac{\mu+\nu}{\beta}=\frac{1}{R_{0}} ; \quad M_{e}=k I_{e}
$$

and $I_{e}$ is implicitly defined as the unique solution of:

$$
1-\frac{1}{R_{0}}-\frac{\mu+\nu}{\mu} I=p_{0}+p_{1}(k I) .
$$

Remark 1. Note that, under the assumption (2.7), by (2.5) and (2.9) it follows that

$$
I \gtreqless I_{e} \quad \Leftrightarrow \quad S^{\prime}\left(S_{e}, I\right) \lesseqgtr 0 .
$$

The local stability of $E E$ depends on the delay parameter $a$ defined in (2.3) via the positivity of the function

$$
f(a)=B_{2} a^{2}+B_{1} a+B_{0} \quad a \in \mathbb{R}_{+}
$$

where

$$
\begin{aligned}
& B_{2}=\beta I_{e}+\mu \\
& B_{1}=\left(\beta I_{e}+\mu\right)^{2}-\beta I_{e} \mu k p_{1}^{\prime}\left(M_{e}\right) \\
& B_{0}=\beta I_{e}\left(\beta I_{e}+\mu\right)(\nu+\mu)
\end{aligned}
$$

The following proposition (see [2]) states our main results on stability.

Proposition 2. If and only if

$$
\left(\beta I_{e}+\mu\right)^{2}-\beta I_{e} \mu k p_{1}^{\prime}\left(k I_{e}\right)+2\left(\beta I_{e}+\mu\right) \sqrt{\beta I_{e}(\nu+\mu)}<0
$$

there exist two values $a_{1}$, $a_{2}$ with $0<a_{1}<a_{2}$ for the parameter a such that EE is unstable for $a \in\left(a_{1}, a_{2}\right)$, whereas it is LAS for a $\notin\left[a_{1}, a_{2}\right]$. At the points $a_{1}$ and $a_{2}$ Hopf bifurcations occur. 


\section{A global bifurcation threshold function}

The condition (2.13) shows that in order to have instability and oscillations it is required that the steepness of the reaction function $p_{1}$ at the endemic equilibrium exceeds a critical threshold suggesting that instability requires a violent (and delayed) reaction by individuals in terms of vaccination coverage to changing epidemiological conditions. Though this interpretation is epidemiologically appealing, it does not need to be correct. Indeed, the quantity $p_{1}^{\prime}\left(k I_{e}\right)$ appearing in condition (2.13) depends on the level of the infective fraction at the equilibrium equilibrium $I_{e}$, which in turn depends on the shape of the $p_{1}$ function through equation (2.9). In this section we attempt at circumventing this difficulty by looking for a suitable reformulation of condition (2.13) having a twofold purpose, i.e. first to also incorporate the role played on stability by the $p_{1}$ function, and second to remove the dependency of $p_{1}$ and its derivative on system parameters. This dependency arises as a consequence of the fact that $p_{1}$ and $p_{1}^{\prime}$ are evaluated at $k I_{e}$ in the standard local stability analysis. This reformulation leads (see (3.18)) to a "global" version of (2.13) in which the values $p_{1}\left(k I_{e}\right)$ and $p_{1}^{\prime}\left(k I_{e}\right)$ are replaced by the functions $p_{1}(x)$ and $p_{1}^{\prime}(x)$ where $x$ takes values in the range of $M$. From a technical point of view this yields a differential inequality in the quantity $q=1-p_{0}-p_{1}$. The solution to such inequality yields a "threshold" function $\phi$ having a clear interpretation: every vaccination function $p_{1}$ always lying below $\phi$, i.e. such that $p_{1}(x)<\phi(x)$ for all admissible values of $x$, will surely lead to stability, i.e. it will never fulfill the condition (2.13), whereas every reaction function $p_{1}$ always lying above $\phi$, will surely lead to instability, i.e. it will always fulfill (2.13). From am epidemiological standpoint this allows to classify the possible forms of the information-dependent vaccination function $p_{1}$ by separating those functional forms always allowing (other things being equal, i.e. given the remaining epidemiological parameters) the local stability of the endemic state from those always leading to instability and steady oscillations about the endemic state. It is to be noticed that this approach only provides a partial ordering between vaccination function. This means that the method is silent for all those vaccination functions that intersect the threshold function $\phi$ in its admissibility set.

In the remaining part of this section we develop our approach. First we derive the above mentioned differential inequality (see (3.18)). Next we solve it by determining the threshold function $\phi$ in closed form. Finally we demonstrate some properties of the threshold function $\phi$ which will prove useful for the subsequent applications. In the subsequent section we illustrate some concrete applications of our approach by comparing its predictions in terms of stability and bifurcation of the endemic state with the results from the standard "local" analysis (i.e. (2.13)) reported in [2].

Since we will focus on the stability/instability condition of the endemic equilibrium we will always work under assumption (2.7), which is necessary and sufficient to its existence.

We start our analysis by explicitly including in the stability/instability condition (2.13) the role of the function $p$. To this aim we note that $I_{e}$ can be implicitly expressed in (2.9) in terms of the value that the coverage function $p$ takes at the equilibrium by

$$
\beta I_{e}=\left(1-p\left(k I_{e}\right)-R_{0}^{-1}\right) R_{0} \mu .
$$

The last relation implies the following (necessary) condition for the existence of $I_{e}$ :

$$
1-p\left(k I_{e}\right)>R_{0}^{-1} \text {. }
$$


Setting for convenience

$$
q(x)=1-p(x)
$$

and

$$
\varepsilon=2 \sqrt{1+\nu / \mu}
$$

using (3.14) and (3.15), we can rewrite (2.13) as

$$
R_{0}^{2} q^{2}\left(k I_{e}\right)+\left(R_{0} q\left(k I_{e}\right)-1\right) k q^{\prime}\left(k I_{e}\right)+\varepsilon R_{0} q\left(k I_{e}\right) \sqrt{R_{0} q\left(k I_{e}\right)-1}<0 .
$$

Since $q\left(k I_{e}\right)>R_{0}^{-1}$, the condition (3.16) is equivalent to

$$
q^{\prime}\left(k I_{e}\right)<-\frac{R_{0} q\left(k I_{e}\right)}{\left(R_{0} q\left(k I_{e}\right)-1\right) k}\left(R_{0} q\left(k I_{e}\right)+\varepsilon \sqrt{R_{0} q\left(k I_{e}\right)-1}\right) .
$$

The inequality (3.17) is still "local", as (2.13), and hence only a direct knowledge of $p$ and consequently of $I_{e}$ allows to draw conclusions on the stability of the endemic state.

Starting from (3.17) our idea is to look for a more restrictive condition that does not depend on the equilibrium value of $I$, i.e. we look for the set of coverage functions that whatever is the value of $I_{e}$ assure the instability or the stability of $E E$.

Formally, we look for solutions of the differential inequality

$$
q^{\prime}(x)<-\frac{R_{0} q(x)}{\left(R_{0} q(x)-1\right) k}\left(R_{0} q(x)+\varepsilon \sqrt{R_{0} q(x)-1}\right)
$$

under condition

$$
R_{0} q(x)>1
$$

Remark 3. The condition (3.19) is less restrictive that it may seem: it means, given that $p$ is an increasing function, that we control the shape of q, i.e. the manner in which it governs the onset of instability, only in the interval where $1-p\left(x_{e}\right)-R_{0}^{-1}$ is strictly positive. Since the endemic level $k I_{e}$ necessarily belongs to this interval our analysis includes all cases that are of practical interest for the analysis of local stability.

We summarize our results by the following:

Proposition 4. The differential inequality (3.18) with the restriction (3.19) has a unique upper solution $\psi$, i.e. a unique function such that any other solution $g$ to (3.18)-(3.19) fullfills $g(x) \leq$ $\psi(x)$. To the upper solution it corresponds a unique and epidemiologically meaningful threshold function $\varphi=1-p_{0}-\psi$ having the following feature: every vaccination function $p_{1}$ always lying below $\varphi$, i.e. such that $p_{1}(x)<\varphi(x)$ for all admissible values of $x$, will surely lead to stability, i.e. it will never fulfill the condition (2.13), whereas every reaction function $p_{1}$ always lying above $\varphi$, will surely lead to instability, i.e. it will always fulfill (2.13). 
We divide the proof in several steps.

To solve (3.18), we look for the solution $\psi: \mathcal{J} \rightarrow\left[R_{0}, 1-p_{0}\right)$, where $\mathcal{J}$ is an interval in $\mathbb{R}$, of the Cauchy problem

$$
\left\{\begin{array}{l}
q^{\prime}=-\frac{R_{0} q}{\left(R_{0} q-1\right) k}\left(R_{0} q+\varepsilon \sqrt{R_{0} q-1}\right) \\
q(0)=1-p_{0}
\end{array}\right.
$$

By the comparison result for ODEs (see [4]) we conclude that all the solutions of (3.18) are functions $g: \mathcal{J} \rightarrow\left[R_{0}, 1-p_{0}\right)$ such that

$$
g(x) \leq \psi(x) \quad \forall x \in \mathcal{J} .
$$

The Cauchy problem (3.20) has a solution (see the Appendix) which can be expressed in implicit form in terms of the function $\phi=1-p_{0}-\psi$ :

$$
\begin{aligned}
x= & \frac{2 k}{\varepsilon} R_{0}^{-1}\left(\arctan \sqrt{Q_{0}-R_{0} \phi(x)}-\arctan \sqrt{Q_{0}}\right)+ \\
& -k R_{0}^{-1} \ln \left(\frac{2 \sqrt{Q_{0}-R_{0} \phi(x)}+\xi}{2 \sqrt{Q_{0}}+\xi}\right)^{1+\eta}\left(\frac{\sqrt{Q_{0}-R_{0} \phi(x)}+2 \xi^{-1}}{\sqrt{Q_{0}}+2 \xi^{-1}}\right)^{1-\eta}
\end{aligned}
$$

where we have set for simplicity (note that $\varepsilon-\widehat{\varepsilon}=4(\varepsilon+\widehat{\varepsilon})^{-1}$ ):

$$
Q_{0}=R_{0}\left(1-p_{0}\right)-1 ; \quad \widehat{\varepsilon}=\sqrt{\varepsilon^{2}-4} ; \quad \xi=\varepsilon+\widehat{\varepsilon} ; \quad \eta=\frac{\varepsilon^{2}-2}{\varepsilon \widehat{\varepsilon}} .
$$

Equation (3.21) defines the required threshold function $\phi$ only implicitly. This does not make its properties immediately evident. Two aspects are worth being investigated. First, in order that $\phi$ represents a meaningful threshold function separating vaccination functions always leading to stability of the endemic state from those always leading to instability, it is necessary to demonstrate that the equilibrium $x_{e}=k I_{e}$ belongs to the domain of $\phi$ for every epidemiologically meaningful parameter values. Moreover, given that the stability/instability of the endemic state depends on the slope of the vaccination function, we need information on the slope of $\phi$. The following Proposition provides the answers to these questions.

Proposition 5. The function $\phi$ is:

1. defined on $\left[0, x_{\max }\right)$, where

$$
x_{\max }=k R_{0}^{-1}\left(-\frac{2}{\varepsilon} \arctan \sqrt{Q_{0}}-\ln 2^{1-\eta} \xi^{2 \eta} \frac{\left(\sqrt{Q_{0}}+2 \xi^{-1}\right)^{\eta-1}}{\left(2 \sqrt{Q_{0}}+\xi\right)^{\eta+1}}\right)
$$

and for all biologically meaningful parameters with $\left(1-p_{0}\right) R_{0}>1$ the equilibrium value $x_{e}=k I_{e}$ belongs to $\left[0, x_{\max }\right)$; 
2. strictly increasing, with

$$
\min _{x \in\left[0, x_{\max }\right)} \phi^{\prime}(x) \geq \frac{2(2+\varepsilon)}{k} .
$$

Proof. Considering in (3.21) $x$ as a function of $y=\varphi(x)$, we obtain the first part of 1. :

$$
\lim _{y \rightarrow\left(1-p_{0}-S_{e}\right)^{-}} x(y)=k R_{0}^{-1}\left(-\frac{2}{\varepsilon} \arctan \sqrt{Q_{0}}-\ln 2^{1-\eta} \xi^{2 \eta} \frac{\left(\sqrt{Q_{0}}+2 \xi^{-1}\right)^{\eta-1}}{\left(2 \sqrt{Q_{0}}+\xi\right)^{\eta+1}}\right)
$$

The second conclusion follows by Remark 1 observing that

$$
\lim _{x \rightarrow x_{\max }} S^{\prime}\left(S_{e}, \frac{x}{k}\right)=-\frac{\beta x_{\max }}{k R_{0}}<0 .
$$

Since $\psi$ solves (3.20) and $\phi^{\prime}=-\psi^{\prime}$ we deduce that $\phi$ is strictly increasing. Furthermore

$$
\phi^{\prime}(0)=\frac{\left(1-p_{0}\right)^{2} R_{0}^{2}}{\left(R_{0}\left(1-p_{0}\right)-1\right) k}+\frac{\varepsilon\left(1-p_{0}\right) R_{0}}{k \sqrt{R_{0}\left(1-p_{0}\right)-1}}
$$

and $\lim _{x \rightarrow x_{\max }} \phi^{\prime}(x)=+\infty$. To study the convexity of $\phi$ it is sufficient to remind that $\phi$ is convex if and only if $\psi$ is concave and by (3.20) to deduce

$$
\psi^{\prime \prime}=-\frac{R_{0} \psi^{\prime}\left(R_{0} \psi-2\right)}{k}\left(\frac{2 R_{0} \psi+\varepsilon \sqrt{R_{0} \psi-1}}{2\left(R_{0} \psi-1\right)^{2}}\right) .
$$

Since $\psi^{\prime}<0$, we have

$$
\psi^{\prime \prime} \gtreqless 0 \quad \Leftrightarrow \quad R_{0} \psi-2 \gtreqless 0 \quad \Leftrightarrow \quad R_{0}\left(1-p_{0}\right)-2 \gtreqless \phi .
$$

We conclude that:

if $1<\left(1-p_{0}\right) R_{0} \leq 2$, then $\phi$ is strictly convex for all $x \in\left[0, x_{\max }\right)$ and

$$
\min _{x \in\left[0, x_{\max }\right)} \phi^{\prime}(x)=\phi^{\prime}(0) \text {. }
$$

If $\left(1-p_{0}\right) R_{0}>2$, by $\phi(0)=0$ and the strictly monotonicity of $\phi$, there exists a unique $x^{\star} \in$ $\left(0, x_{\max }\right)$ such that $\phi$ is strictly concave on $\left[0, x^{\star}\right]$ and strictly convex on $\left[x^{\star}, x_{\max }\right)$ and, by $\phi\left(x^{\star}\right)=$ $1-p_{0}-2 R_{0}^{-1}$, we obtain

$$
\min _{x \in\left[0, x_{\max }\right)} \phi^{\prime}(x)=\phi^{\prime}\left(x^{\star}\right)=\frac{2(2+\varepsilon)}{k} .
$$

Finally, since for any value of the parameters (with $1-p_{0}-R_{0}^{-1}>0$ )

$$
\phi^{\prime}(0) \geq \frac{2(2+\varepsilon)}{k}
$$

the validity of (3.23) follows. 


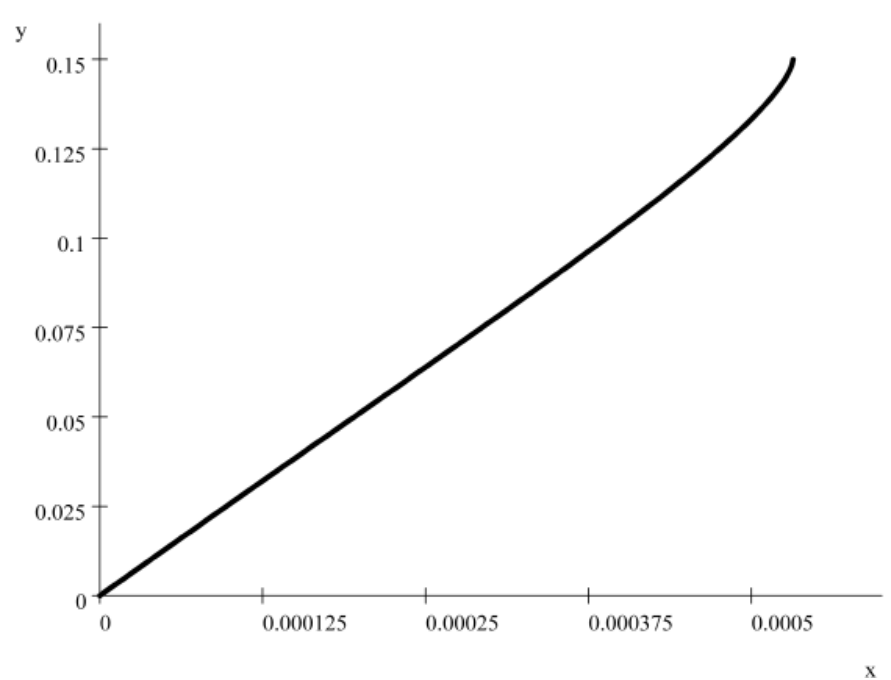

Figure 1: Graph of $\phi$ for $k=1, R_{0}=10, p_{0}=0.75, \nu=7^{-1} *$ days $^{-1}, \mu=(365 \cdot 75)^{-1} *$ days $^{-1}$.

An example of $\phi$ is illustrated in Figure 1, where it is plotted by using the following parameter values roughly mimicking measles in developed countries: mean duration of the disease $\nu^{-1}=1$ week, life expectancy of the host $\mu^{-1}=75$ years, basic reproduction number $R_{0}=10$. Moreover we took $k=1$, and baseline vaccination $p_{0}=0.75$. We shall use these values in the next examples and figures.

The result obtained by solving the differential inequality (3.18) have a nice interpretation in terms of the graph of the $\phi$ function. The function $\phi$ plays the role of a stability "threshold line". In fact reaction functions $p_{1}$ (obeying assumptions A1)-A3)) whose graph lie below the graph of $\phi$ for all $x \in\left[0, x_{\max }\right)$ represent vaccination behaviours that lead to the stability of endemic state. On the other hand, coverage functions whose graphs lie above $\phi$ for any admissible $x$ will lead, for properly chosen parameter constellations, to instability and oscillations.

The shape of the threshold function $\phi$ obviously depends on the values of the system parameters appearing in the "global" stability condition (3.18) Figure 2 shows the sensitivity of the threshold function to changes in the basic reproduction number $R_{0}$.

A further aspect concerns the difficulty to analitically compare a coverage function with $\phi$ since this last is only implicitly defined. With regard to this, we note that (3.23) allows us to state a much simpler stability test: given any reaction function $p_{1}$, if

$$
p_{1}^{\prime}(x)<\frac{2(2+\varepsilon)}{k} \quad \forall x
$$

then $p_{1}(x)<\phi(x)$ for all $x \in\left(0, x_{\max }\right)$.

Note that unfortunately it does not appear to be possible to find an instability test of simple application as (3.24). 


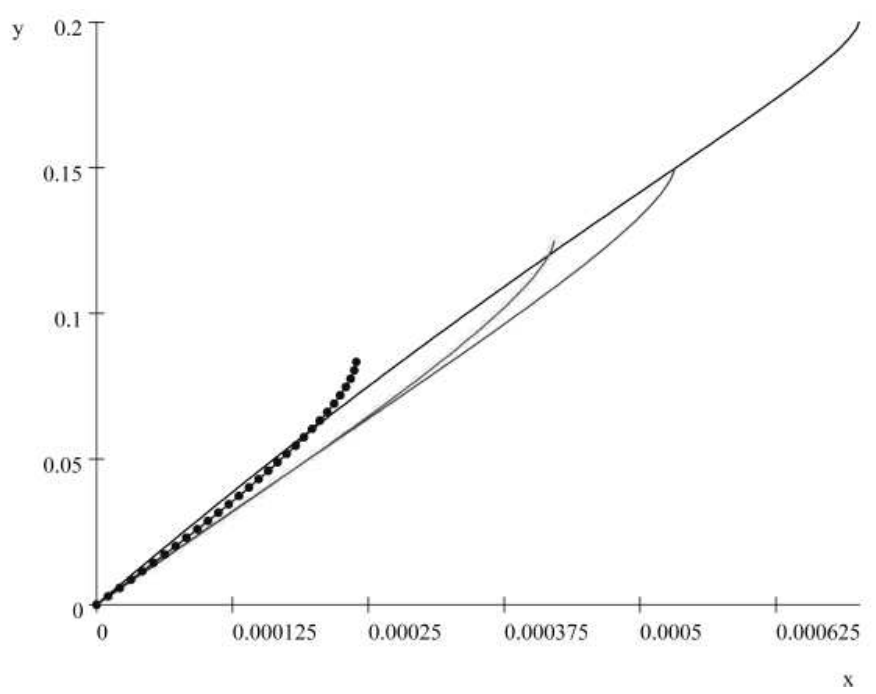

Figure 2: Sensitivity analysis of $\phi$ with respect to $R_{0}$ in the cases $R_{0}=20$ (continue line), $R_{0}=10$ (dashed line), $R_{0}=8$ (dotted line) and $R_{0}=6$ (squares).

\section{Examples}

The following examples based on classic functional forms of $p_{1}$ illustrate all the aspects of the previous discussion.

Example 6. (The piece-wise linear case) A useful, though not fully realistic, functional form is

$$
p_{1}(x)=\min \left\{c x, 1-p_{0}\right\} \quad x \in \mathcal{I}, c \in \mathbb{R}_{+} .
$$

Since $p_{1}^{\prime}(x)=$ cfor all $x \in\left[0,\left(1-p_{0}\right) c^{-1}\right)$ and $\phi(x)<1-p_{0}$, by (3.23) to assure $p_{1}(x)<\phi(x)$ for all $x \in\left[0, x_{\max }\right)$ is sufficient that

$$
c<\frac{2(2+\varepsilon)}{k} .
$$

For example, with the above values of the parameters, we have that it must be $0<c<c^{*}$ where $c^{*} \simeq 254.175$. This is illustrated in Figure 3, where we have plotted both $\phi$ and $p_{1}$ for $c=200<c^{*}$ and $c=350>c^{*}$.

The previous results are consistent with the "local" investigation of the bifurcation condition, which is illustrated in Figure 4 reporting the shape of the function $f$ that determines stability, as a function of the delay parameter a, for various values of c. Figure 4 shows that stability is lost for values of c around 257, which are lower estimated by our global condition.

This example also shows the difficulty to state a sufficient condition of instability in the same spirit of (4.25). Any function $p_{1}$ intersecting $\phi$ represents an ambiguous case from our "global" perspective; Figure 5 illustrates this circumstance: since p does not always lie below (above) the threshold function $\phi$, our approach does not supply useful information. In this case the stability 


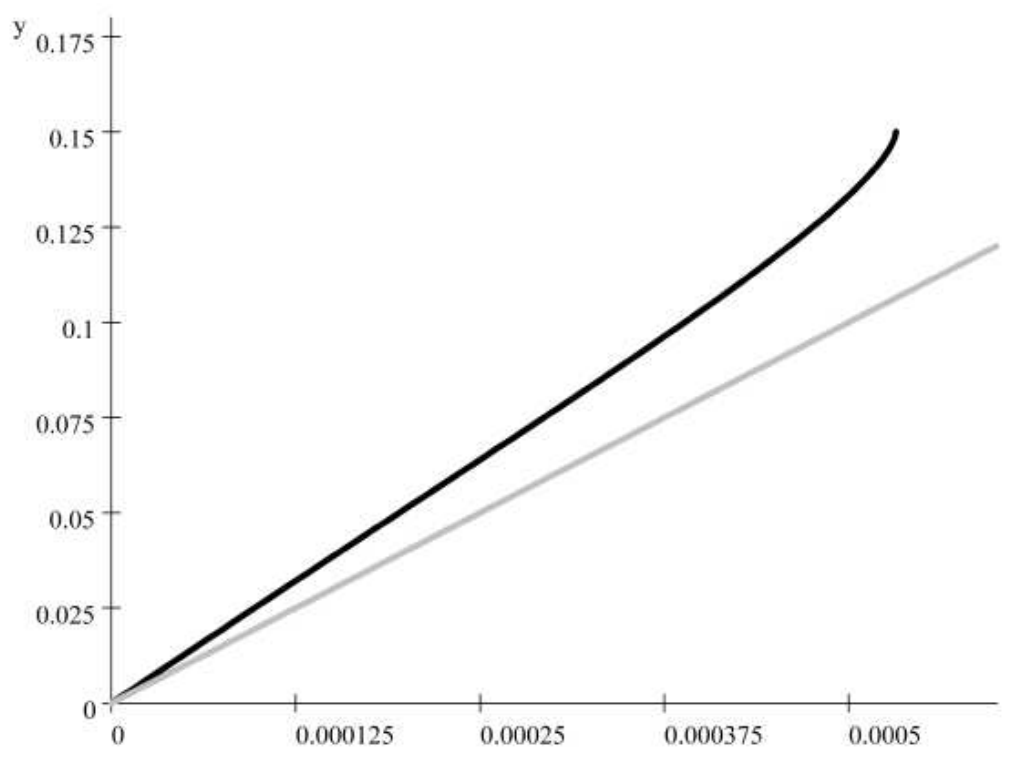

(a)

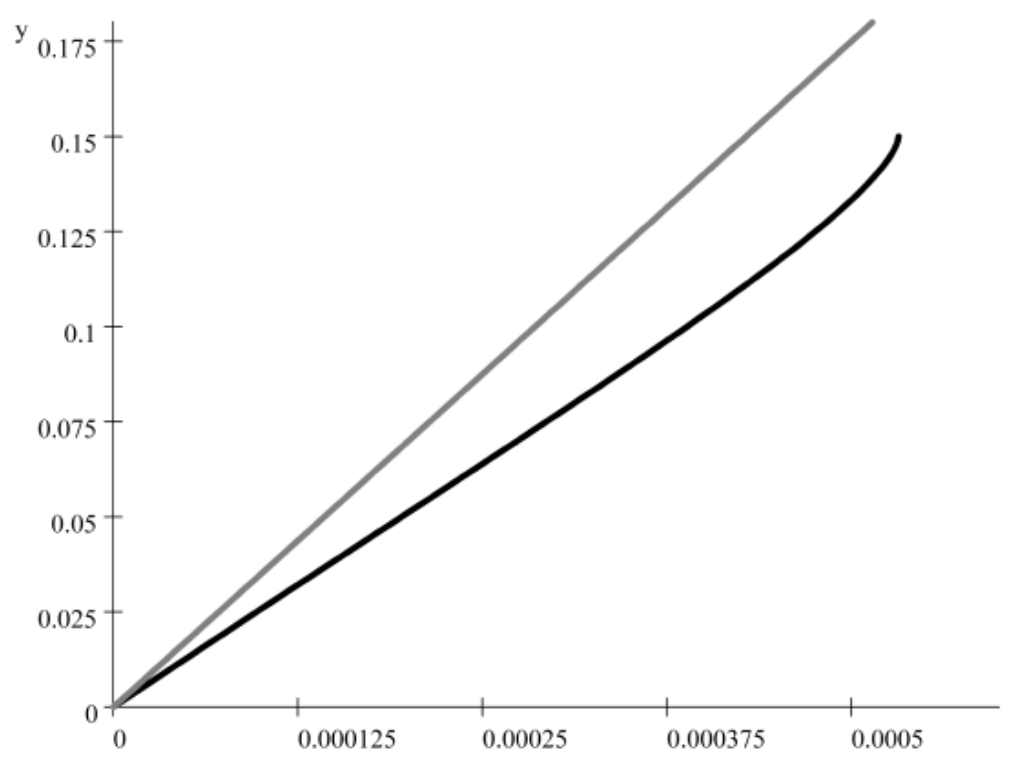

(b)

$\mathrm{x}$

Figure 3: The piece-wise linear case: (a) stability case $\left(\phi>p_{1}\right)$ for $p_{0}=0.75 ; R_{0}=10 ; k=$ $1 ; c=200$; (b) instability case $\left(\phi<p_{1}\right)$ for $p_{0}=0.75 ; R_{0}=10 ; k=1 ; c=350$. 


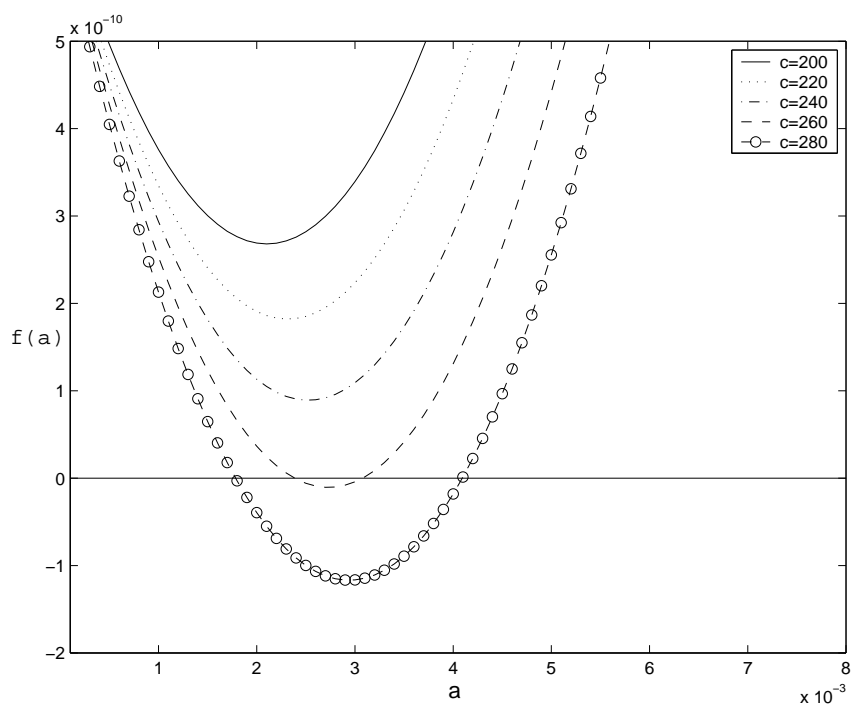

Figure 4: The piece-wise linear case: shape of $f(a)$ for different values of $c$ from $c=200$ (higher curve) to $c=280$ (lower curve).

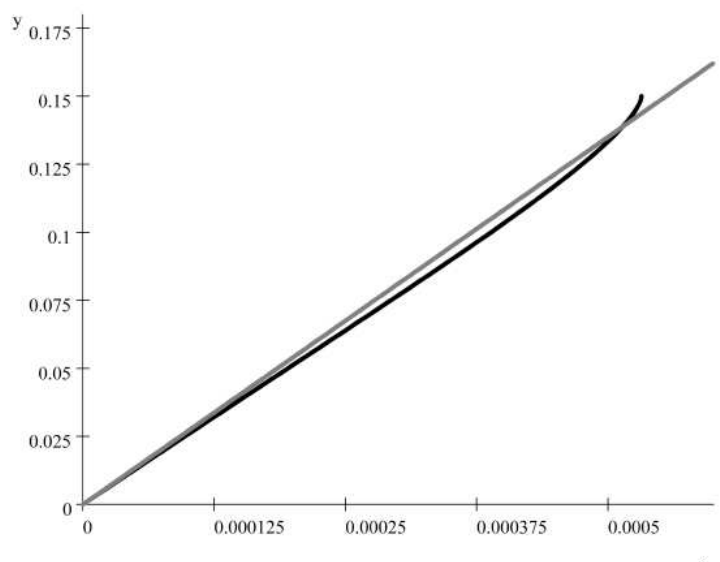

Figure 5: The piece-wise linear case: an ambiguous case ( $\left.p_{0}=0.75 ; R_{0}=10 ; k=1 ; c=270\right)$. 
can be detected by the "local" but conclusive condition (2.13), but since p is not comparable with $\phi$ no conclusions can be drawn from our analysis.

Example 7. (The Michaelis-Menten-Monod case). A more biologically founded functional form is the Michaelis-Menten-Monod function (see [9]), given by

$$
p_{1}(x)=\frac{C x}{D x+1} \quad C, D \in \mathbb{R}_{+} .
$$

The previous function is concave with $C=p_{1}^{\prime}(0)$. This implies:

$$
p_{1}(x) \leq C x \quad \forall x \in\left[0, x_{\max }\right) .
$$

By the linear case it follows that, if

$$
C<\frac{2(2+\varepsilon)}{k}, \quad \forall D \in \mathbb{R}_{+}
$$

the stability condition is satisfied. We note that as in the piece-wise linear case, with respect to the parameter constellation we are considering, the exact stability/instability value $C^{*} \simeq 280.32$ (corresponding to $D^{*} \simeq 1168$, see [2]) is roughly estimated by $2(2+\varepsilon) / k=254.175$.

Example 8. (The Holling type 2 case, see [9]). Another function which probably better captures apriori guesses about $p_{1}$ is:

$$
z(M)=\frac{C M^{2}}{D M^{2}+1} \quad M \in\left[0, M_{\max }\right)
$$

with $C$ and $D$ in $\mathbb{R}_{+}$with $1-p_{0} \geq C / D$. In phenomenological terms this function implies a response of vaccination to changing epidemiological conditions which is quite flat for very low levels of disease prevalence, then starts to climb very quickly, when diseases prevalence goes above some reference level, and finally saturates. The function $z$ has the first two derivatives

$$
z^{\prime}(M)=\frac{2 C M}{\left(D M^{2}+1\right)^{2}} ; \quad z^{\prime \prime}(M)=\frac{2 C\left(1-3 D M^{2}\right)}{\left(D M^{2}+1\right)^{3}} .
$$

Since $z(0)=\varphi(0)=0$ with $z^{\prime}(0)=0<\varphi^{\prime}(0)$, and the maximum of $z^{\prime}$ is reached in $M^{*}=$ $\sqrt{1 / 3 D}$ with

$$
z^{\prime}\left(M^{*}\right)=\frac{9 C}{8 \sqrt{3 D}}
$$

a sufficient condition of stability is then

$$
C \leq \min \left\{\frac{16(2+\varepsilon) \sqrt{3 D}}{9 k},\left(1-p_{0}\right) D\right\} .
$$

For example, in Figure 7, for $p_{0}=0.75 ; R_{0}=10 ; k=1$ the region of stability in the plane of the parameters $C$ and $D$ is the region between the curve plotted and the $D$ axis. 


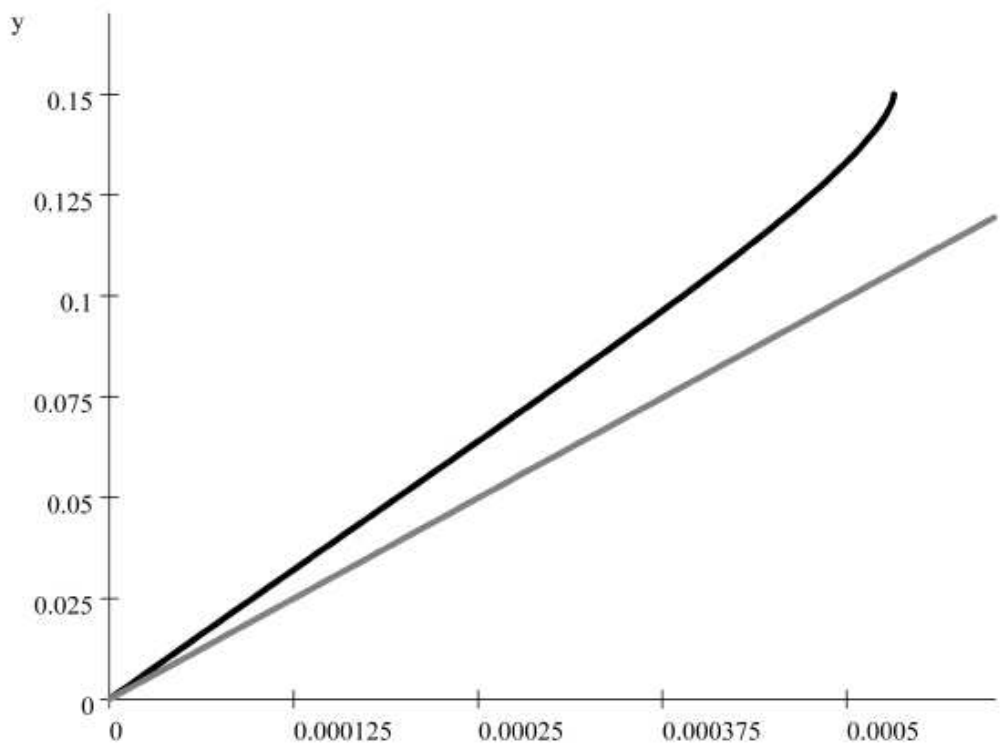

(a)

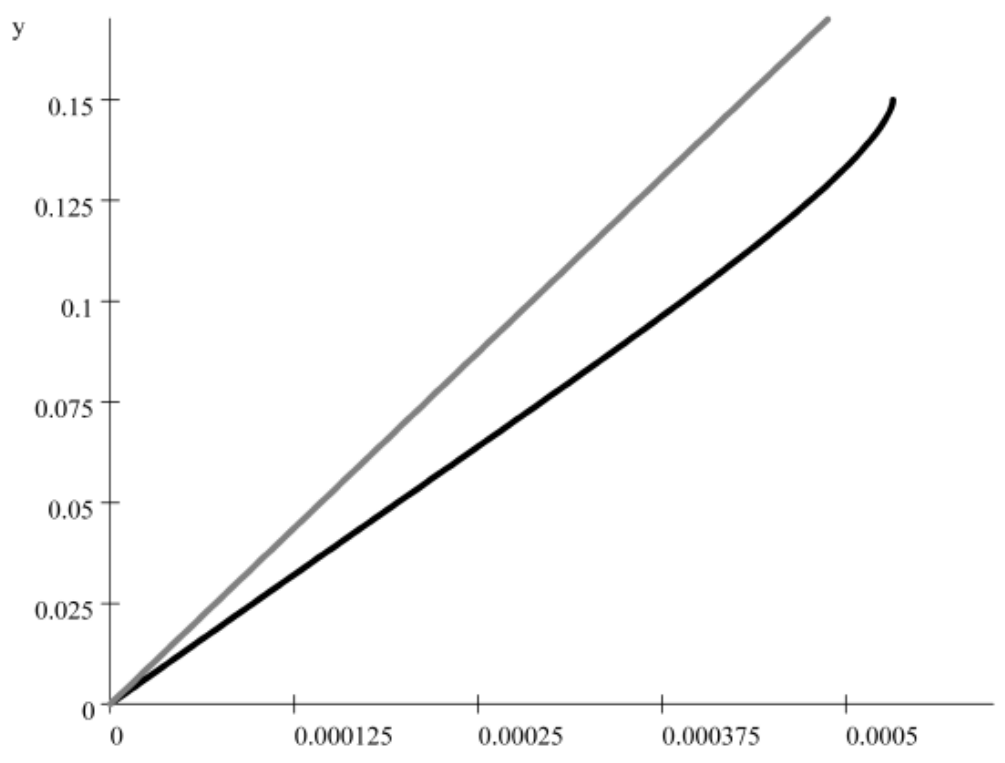

(b)

Figure 6: The Michaelis-Menten-Monod case: (a) stability case $\left(\phi>p_{1}\right)$ for $p_{0}=0.75 ; R_{0}=$ $10 ; k=1 ; C=200 ;$ (b) instability case $\left(\phi<p_{1}\right)$ for $p_{0}=0.75 ; R_{0}=10 ; k=1 ; C=350$. 


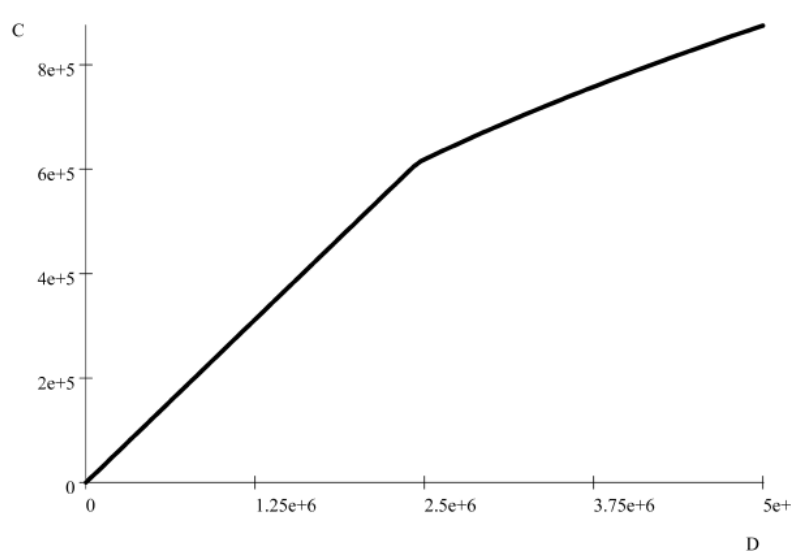

Figure 7: The Holling type 2 case: graph of $\min \left\{\frac{16(2+\varepsilon) \sqrt{3 D}}{9 k},\left(1-p_{0}\right) D\right\}$.

\section{Appendix}

Setting for simplicity $z=R_{0} q$, we have to solve:

$$
\int_{R_{0}\left(1-p_{0}\right)}^{z} \frac{u-1}{u(u+\varepsilon \sqrt{u-1})} d u=-\frac{R_{0}}{k} x .
$$

Setting $t=\sqrt{u-1}$ i.e. $u=t^{2}+1$, the integral in (5.26) is equivalent to

$$
\int_{\sqrt{R_{0}\left(1-p_{0}\right)-1}}^{\sqrt{z-1}} \frac{2 t^{3}}{\left(t^{2}+1\right)\left(t^{2}+\varepsilon t+1\right)} d t
$$

The discriminant

$$
\Delta=\varepsilon^{2}-4=4 \frac{\nu}{\mu}
$$

of the second order polynomial $t^{2}+\varepsilon t+1$ is positive, hence we can factorize it as:

$$
t^{2}+\varepsilon t+1=\left(t+\frac{\varepsilon}{2}+\frac{\widehat{\varepsilon}}{2}\right)\left(t+\frac{\varepsilon}{2}-\frac{\widehat{\varepsilon}}{2}\right)
$$

where $\widehat{\varepsilon}=\sqrt{\varepsilon^{2}-4}=2 \sqrt{\nu / \mu}$. Since

$$
\frac{2 t^{3}}{\left(t^{2}+1\right)\left(t^{2}+\varepsilon t+1\right)}=-\frac{2}{\varepsilon\left(t^{2}+1\right)}+\frac{\varepsilon^{2}+\varepsilon \widehat{\varepsilon}-2}{\varepsilon \widehat{\varepsilon}\left(t+\frac{\varepsilon}{2}+\frac{\widehat{\varepsilon}}{2}\right)}+\frac{\varepsilon \widehat{\varepsilon}-\varepsilon^{2}+2}{\varepsilon \widehat{\varepsilon}\left(t+\frac{\varepsilon}{2}-\frac{\widehat{\varepsilon}}{2}\right)}
$$

the primitive of $2 t^{3}\left(t^{2}+1\right)^{-1}\left(t^{2}+\varepsilon t+1\right)^{-1}$ is

$$
-\frac{2}{\varepsilon} \arctan (t)+\left(1+\frac{\varepsilon^{2}-2}{\varepsilon \widehat{\varepsilon}}\right) \ln \left(t+\frac{\varepsilon}{2}+\frac{\widehat{\varepsilon}}{2}\right)+\left(1-\frac{\varepsilon^{2}-2}{\varepsilon \widehat{\varepsilon}}\right) \ln \left(t+\frac{\varepsilon}{2}-\frac{\widehat{\varepsilon}}{2}\right)
$$


and (5.27) is equal to

$$
\begin{aligned}
& -\frac{2}{\varepsilon}\left(\arctan (\sqrt{z-1})-\arctan \left(\sqrt{R_{0}\left(1-p_{0}\right)-1}\right)\right)+ \\
& +\left(1+\frac{\varepsilon^{2}-2}{\varepsilon \widehat{\varepsilon}}\right) \ln \left(\frac{2 \sqrt{z-1}+\varepsilon+\widehat{\varepsilon}}{2 \sqrt{R_{0}\left(1-p_{0}\right)-1}+\varepsilon+\widehat{\varepsilon}}\right)+ \\
& +\left(1-\frac{\varepsilon^{2}-2}{\varepsilon \widehat{\varepsilon}}\right) \ln \left(\frac{2 \sqrt{z-1}+\varepsilon-\widehat{\varepsilon}}{2 \sqrt{R_{0}\left(1-p_{0}\right)-1}+\varepsilon-\widehat{\varepsilon}}\right)
\end{aligned}
$$

\section{Acknowledgements}

The authors thank an anonymous reviewer of the Journal whose suggestions significantly improved the clarity of the exposition.

\section{References}

[1] R.M. Anderson, R.M. May. Infectious diseases of humans: dynamics and control, Oxford University Press, Oxford, 1998.

[2] A. D'Onofrio, P. Manfredi, E. Salinelli. Vaccinating behavior, information, and the dynamics of SIR vaccine preventable diseases. Theoretical Population Biology, in press (2006), available online at http://ees.elsevier.com/tpb/.

[3] J. Guckenheimer, P. Holmes. Nonlinear oscillations, dynamical systems and bifurcation of vector fields. Springer Verlag, New York, 1983.

[4] J.K. Hale. Ordinary differential equations. Wiley and Sons, 1969.

[5] W. Liu. Dose-dependent latent period and periodicity of infectious diseases. J. Math. Biol. 31 (1993), 487-494

[6] E.T. Luman, A.E. Fiore, T.W. Strine, L.E. Barker. Impact of thimerosal-related changes in hepatitis B vaccine birth-dose recommendations. J. Amer. Med. Ass., 291 (2004), 2351-2358.

[7] A. Maayan-Metzger, P. Kedem-Friedrich, J. Kuint. To vaccinate or not to vaccinate - that is the question: why are some mothers opposed to giving their infants hepatitis $B$ vaccine? Vaccine, 23 (2005), 1941-1948. 
[8] N. MacDonald. Biological delay systems: linear stability theory. Cambridge University Press, 1989.

[9] J.D. Murray. Mathematical biology. Springer Verlag, New York, 1989.

[10] T.C. Reluga, C.T. Bauch, A.P. Galvani. Evolving public perceptions and stability in vaccine uptake. Mathematical Biosciences, in press (2006).

[11] D.A.Salmon, S.P. Teret, C.R. MacIntyre, D. Salisbury, M.A. Burgess, N.A. Halsey. Compulsory vaccination and conscientious or philosophical exemptions: past, present and future. The Lancet, 367 (2006), 436-442.

[12] J.P. Stiglitz. Economics of the public sector. W.W. Norton \& Company, 2000.

[13] H. Thieme. Mathematics in population biology. Princeton University Press, Princeton, 2003.

[14] WHO 2006, Site on immunization safety, http://www.who.int/immunization_safety/aefi/en/.

[15] J.A. Wright, C. Polack. Understanding variation in measles-mumps-rubella immunization coverage-a population-based study. Eur. J. Public Health, 16 (2005), No. 2, 137-142. 